\title{
Pediatric ophthalmology and strabismus: something for everyone.
}

Alex V. Levin

Wills Eye Institute, Thomas Jefferson University

Follow this and additional works at: https://jdc.jefferson.edu/willsfp

Part of the Ophthalmology Commons

Let us know how access to this document benefits you

\section{Recommended Citation}

Levin, Alex V., "Pediatric ophthalmology and strabismus: something for everyone." (2010). Wills Eye Hospital Papers. Paper 5.

https://jdc.jefferson.edu/willsfp/5

This Article is brought to you for free and open access by the Jefferson Digital Commons. The Jefferson Digital Commons is a service of Thomas Jefferson University's Center for Teaching and Learning (CTL). The Commons is a showcase for Jefferson books and journals, peer-reviewed scholarly publications, unique historical collections from the University archives, and teaching tools. The Jefferson Digital Commons allows researchers and interested readers anywhere in the world to learn about and keep up to date with Jefferson scholarship. This article has been accepted for inclusion in Wills Eye Hospital Papers by an authorized administrator of the Jefferson Digital Commons. For more information, please contact: JeffersonDigitalCommons@jefferson.edu. 


\title{
As submitted: \\ Current Opinion in Ophthalmology
}

And later published as:

"Pediatric ophthalmology and strabismus: something for
everyone"

\author{
Alex V. Levin \\ Volume 21, Issue 5, September 2010, Pages 327-328 \\ DOI: $10.1097 /$ ICU.0b013e32833cd438
}

Pediatric Ophthalmology and Ocular Genetics, Wills Eye Institute, Thomas Jefferson University, Philadelphia, Pennsylvania, USA

Correspondence to Alex V. Levin, MD, MHSc, Chief, Pediatric Ophthalmology and Ocular Genetics, Wills Eye Institute, Suite 1210, 840 Walnut Street, Philadelphia, PA 19107-5109, USA Tel: +1 215928 3914; fax: +1 215928 3983; e-mail:

alevin@willseye.org

Current Opinion in Ophthalmology 2010, 21:327-328

This issue of Current Opinion in Ophthalmology highlights the uniquely broad range of pediatric ophthalmology and strabismus. Perhaps, unlike any other area of ophthalmology, our specialty covers virtually every part of the eye and a multitude of systemic disorders. Pediatric ophthalmology offers the practitioner the opportunity to impact a patient's visual life for many decades giving them a lifetime of good vision. This issue of the journal highlights the many ways that this is so.

As survival increases for babies born prematurely around the world, the challenge of retinopathy of prematurity (ROP) is growing. As one of the leading causes of blindness in children worldwide, ROP has become one area of pediatric ophthalmology that has received extraordinary research attention in developing treatment protocols, at first cryotherapy, and now laser ablation of avascular retina. The future focus will be on refining our treatment strategies, providing care in the third world where survival of 
premature babies is on the rise but ophthalmic care may be less available, the use of novel strategies for treatment such as intravitreal antivasoproliferative factors, identification of genetic predisposition to the disease, and the use of imaging techniques to improve screening. The field of ROP continues to be the highest risk in terms of malpractice in all of ophthalmology. This situation has led many physicians to turn away from the care of these babies. Yet, as highlighted by Salvin et al. (pp. 329-334), excellent systems for identification, following and treatment of these children exist and allow pediatric ophthalmologists, and retinal specialists to feel better protected in their care of these children. In addition to ROP we must not forget the other ocular manifestations of prematurity including high myopia, strabismus, and cortical visual impairment.

Strabismus is the 'bread and butter' of most pediatric ophthalmic practices. Nelson and Wang (pp. 335-340) remind us that new approaches to this disorder are continuously being investigated. Children with strabismus suffer great psychosocial disadvantages and the same remains true even in adulthood. Strabismus surgery is often the first encounter that an ophthalmic resident has with surgical intervention and this is another reason why it is a foundational aspect of the specialty of ophthalmology. In virtually, every subspecialty in ophthalmology, patients will present with strabismus that needs attention. As discussed, by Gunton (pp. 341-344), diplopia can be disabling in adults following cataract extraction and refractive surgery. The recognition of specific disorders such as elevation deficieny and convergence insufficiency, reviewed by Manley and Lavrich (pp. 356-360) respectively, further our ability to deliver exacting care to these patients in need. And like any surgical intervention, one must be cognizant of the complications of surgery along with their management as Simon (pp. 361-366) informs us.

Cataract surgery in children remains challenging in terms of identification of patients who may benefit from surgery, the procedure itself, and long-term follow-up. Intraocular lens (IOL) use in children was introduced without any randomized prospected studies. Rather, this innovation came as a theoretical application from adult successes. Although, IOL have benefited many children and prevented the cost and inconvenience of contact lens wear, it has yet to be shown clearly that IOL gives a better visual outcome as compared with contact lens. This is particularly true in infants where the complication rate of IOL surgery is quite concerning and is currently the subject of a large prospective multicenter trial in the field. Perhaps the most difficult aspect of IOL implantation in children is, knowing when not to do it. Infants and children with uveitis, microphthalmia, and eyes that have failed patching for ambloypia all require extra attention and thought before proceeding with surgery. Just because we can now technically implant an IOL in almost any eye, does it mean it is the right thing to do in every case? Hug (pp. 345-349) elegantly discusses some of these challenges.

The concept of refractive surgery in childhood, as reviewed by Wasserman (pp. 350355), remains quite controversial. The long-term effects and outcomes for children remain largely unknown. This factor in turn leaves us with the puzzle of recognizing the appropriate indications. In addition, many children will require general anesthesia for this procedure, which, although usually with minimal risk, still requires a proper riskbenefit assessment before proceeding. Children with anesotropia and patching failure 
may benefit. Clearly, further research is indicated, preferably in a prospective fashion, to allow us to best identify the appropriate indications for pediatrics.

Lastly, Brian Forbes (pp. 367-374) discusses just one of the many systemic associations that bring children to the care of their pediatric ophthalmologist. Craniofacial abnormalities are rare but fascinating and present many challenges for the pediatric ophthalmologist including the management of strabismus, ocular complications of their craniofacial surgery, optic nerve compression, and the associated oculofacial and ophthalmic malformations. Children with these disorders benefit from care in a focused multidisciplinary setting by providers who have familiarity and expertise with these uncommon disorders. Pediatric ophthalmologists consistently deal with the ocular manifestations of rheumatologic, hematologic, neurologic, metabolic and genetic diseases. This factor is one of the many beauties of our specialty.

Each year in North America, there are more pediatric ophthalmology fellowship positions offered than applicants. Pediatric ophthalmology is one of the smaller subspecialties in our field. It is also known for its lower remuneration. Yet every day, pediatric ophthalmologists are faced with the possibility of seeing an enormous range of ophthalmic manifestations affecting virtually every part of the eye. Pediatric ophthalmologists do glaucoma, cataract, oculoplastics, and in some cases, even corneal surgery. What a wonderful opportunity for a budding ophthalmologist! Although we sometimes see the tragedy of childhood illness and visual loss, and occasionally the impact of broken or abusive families, there is a special joy in helping a child and their family. Most often, each child comes with a caretaker, to whom that child is the most important person in the world. What a wonderful partner in the care of our patients.

I hope that this issue, presented to you by authors all from the Wills Eye Institute family, current staff and graduates of our programs, will entice residents to join our special field, general ophthalmologist to see more pediatric patients, and further enhance what I feel, is the best subspecialty in ophthalmology! 